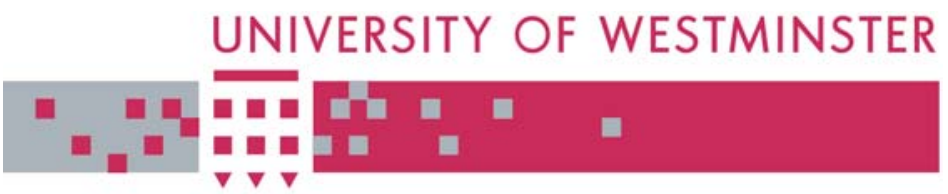

\title{
WestminsterResearch
}

http://www.wmin.ac.uk/westminsterresearch

\section{A system for patient management based discrete-event simulation and hierarchical clustering.}

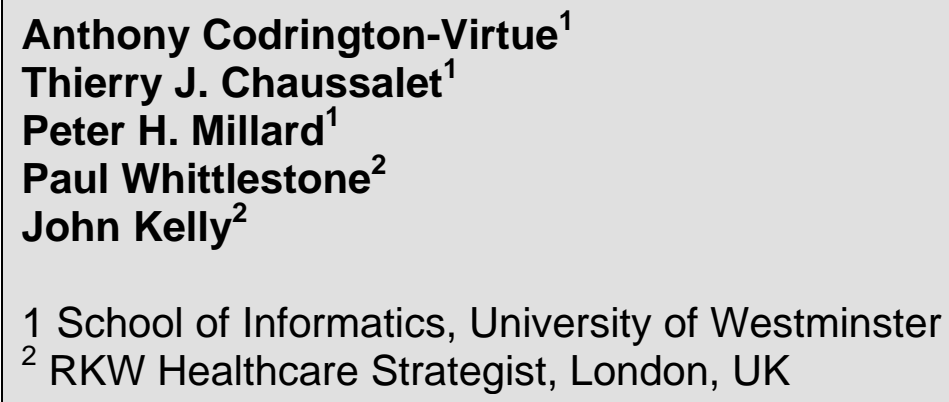

Copyright (c) [2006] IEEE. Reprinted from Nineteenth IEEE International Symposium on Computer-Based Medical Systems: 22-23 June June 2006, Salt Lake City, Utah. Proceedings, pp. 800-804.

This material is posted here with permission of the IEEE. Such permission of the IEEE does not in any way imply IEEE endorsement of any of the University of Westminster's products or services. Internal or personal use of this material is permitted. However, permission to reprint/republish this material for advertising or promotional purposes or for creating new collective works for resale or redistribution must be obtained from the IEEE by writing to pubs-permissions@ieee.org. By choosing to view this document, you agree to all provisions of the copyright laws protecting it.

The WestminsterResearch online digital archive at the University of Westminster aims to make the research output of the University available to a wider audience. Copyright and Moral Rights remain with the authors and/or copyright owners.

Users are permitted to download and/or print one copy for non-commercial private study or research. Further distribution and any use of material from within this archive for profit-making enterprises or for commercial gain is strictly forbidden.

Whilst further distribution of specific materials from within this archive is forbidden, you may freely distribute the URL of the University of Westminster Eprints (http://www.wmin.ac.uk/westminsterresearch).

In case of abuse or copyright appearing without permission e-mail wattsn@wmin.ac.uk. 


\title{
A system for patient management based discrete-event simulation and hierarchical clustering
}

\author{
Anthony Codrington-Virtue ${ }^{1}$, Thierry Chaussalet ${ }^{1}$, Peter Millard ${ }^{1}$, \\ Paul Whittlestone ${ }^{2}$, John Kelly ${ }^{2}$ \\ ${ }^{1}$ Health and Social Care Modelling Group, University of Westminster, London, UK \\ ${ }^{2} R K W$ Healthcare Strategist, London, UK \\ \{codrina,chausst\}@wmin.ac.uk,phmillard@tiscali.co.uk, \\ \{paul.whittlestone,john.kelly\}@rkw-uk.com
}

\begin{abstract}
Hospital Accident and Emergency (A\&E) departments in England have a 4 hour target to treat $98 \%$ of patients from arrival to discharge, admission or transfer. Managing resources to meet the target and deliver care across the range of $A \& E$ services is a huge challenge for $A \& E$ managers. This paper develops an intelligent patient management tool to help managers and clinicians better understand patient length of stay and resources within an $A \& E$ area. The developed discrete-event simulation model gives a highlevel representation of ambulance arrivals into $A \& E$. The model facilitates analysis in the following ways: visually interactive software showing patient length of stay in the $A \& E$ area; patient activity broken down into sub-groups so that intelligence might be gathered on how sub-groups affect the overall length of stay; understanding the number of patient treatment places and nurse resources required. To support ease of inputs for scenario and sensitivity testing, data is entered into the simulation model (Simul8) via Excel spreadsheets. The model discussed in this paper used patient length of stay grouped by $A \& E$ diagnosis codes and was limited to ambulance arrivals. The analysis was derived from A\&E attendance in 2004 from an English hospital.
\end{abstract}

\section{Introduction}

Accident and Emergency (A\&E) departments in the National Health Service (NHS) in England are constantly under pressure to meet the ever increasing demands of providing health care. A key target is that $98 \%$ of patients should be discharged, admitted or transferred within 4 hours of arrival at A\&E [1]. One of the challenges for managers is how to make the best use of resources. Health care has numerous examples of application of operational research techniques, such as discrete-event simulation, to help managers better understand patient activity [2], [3], [4], [5]. Walczak et al. [6] used data set variables, groupings defined by domain experts and neural networks as a tool to derive hospital bed resources in an intensive care unit. Isken et al. [7] discussed the use of data mining and Kmeans clustering to model obstetrics and gynecological patient flows in hospital. This paper proposes a system for intelligent patient management, looking at clustered patient sub-groups, patient treatment place resources and nurse resources in $\mathrm{A} \& \mathrm{E}$, to facilitate improvements in patient care. This paper will take a high level overview of $A \& E$ ambulance arrivals and derive patient length of stay clustered by patient diagnosis codes. The clustered length of stay were further analysed to determine the arrival pattern of the clustered group. Competing resources such as the number of treatment places and nurses were modelled using clustered groups and the associated clustered arrival pattern. In this way, we compared clustered groups with long lengths of stay (with their associated arrival pattern and resources) with clustered groups with shorter lengths of stay. The system described in this paper uses Excel spreadsheets to drive a visually interactive discrete-event simulation software package SIMUL8 [8]. The spreadsheets act as interfaces to facilitate ease and speed of data entry to assist the high level overview. The number of clustered groups was set to three, however, the number of groups can be increased for greater detail. This study focuses on ambulance hospital arrivals in 2004 at a English hospital, using patient data from arrival to discharge. For the model, all admissions and transfers were treated as discharges. The following section will briefly give an overview of the A\&E clinical practice of the hospital in this study, in conjunction with the simulation model. 


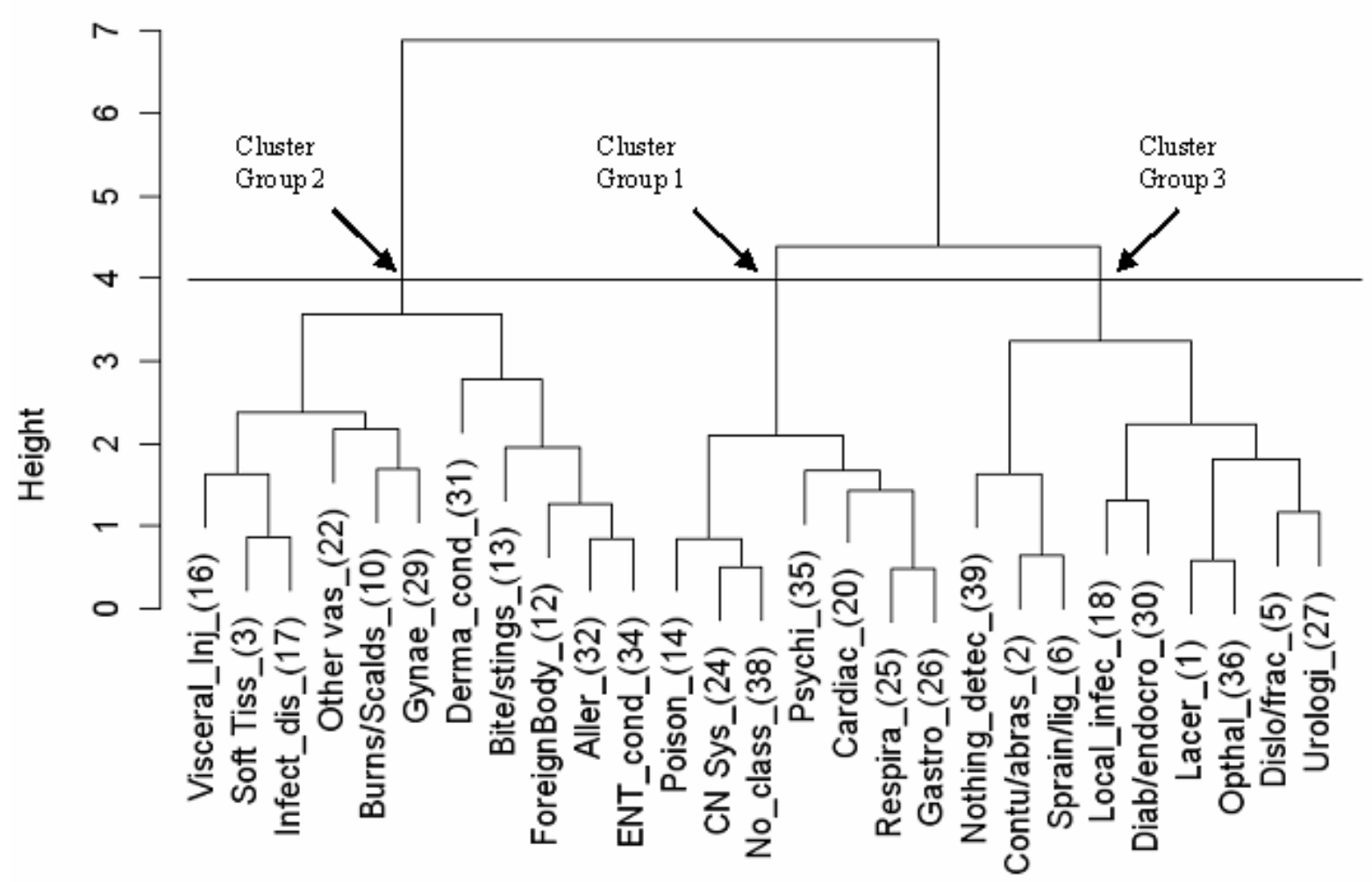

Figure 1. Cluster of 2004 ambulance arrivals by patients length of stay using diagnosis codes defined by A\&E Commissioning Data Sets.

\section{Simulation Model of the A\&E Department}

The simulation model has two modes of arrival into A\&E. Patients either walk in (ambulant) or arrive via an ambulance. On arrival into A\&E, patients are treated in three main areas: the minor injuries unit; the rapid assessment unit (also known as major injuries); and the resuscitation unit (also known as resus). Ambulance arrivals move either into rapid assessment or resuscitation. Whereas, walk-in arrivals pass through nurse led triage, into minor injuries or rapid assessment. Within the model, adults and paediatrics (children) have separate treatment areas within the minor injuries and rapid assessment units. Adult rapid assessment is further subdivided into two treatment areas: Medicine, and Surgery and Orthopaedics.

After triage, patients are assigned a nurse until their discharge from A\&E. The time from patient arrival to discharge is defined here as the length of stay. Furthermore, in the context of this paper, the length of stay includes all the supporting A\&E treatments and activities, such as radiology, blood sampling etc. In practice, nurses are often assigned to more than one patient. For instance, a nurse working within minor injuries might be assigned up to five or six different patients at a time. Whilst a nurse working in rapid assessment might be assigned up to four or five patients at a time and a nurse working in resuscitation might be assigned one or two patients. Similarly, doctors are often assigned to one or more patients. For doctors a typical ratio might be three or four patients for every doctor.

Specifically assigned triage nurses (typically two or three nurses) triage minor patient arrivals. In contrast, triage for ambulance arrivals is performed by the ambulance service. Therefore, in the model, rapid assessment and resuscitation patients would have nurses and treatment places assigned to them on arrival and remain assigned throughout their length of stay in A\&E. Whereas, for minor injuries, nurses are typically assigned patients after they have passed through triage. Similarly, treatment places are allocated to minor patients after triage. However, both nurses and treatment places are limited resources and can only be allocated if available.

\section{Patient Data, Clusters and Arrival patterns}

On arrival at A\&E and during their stay, patients' information is recorded using codes defined by Accident and 
Table 1. Quantile percentages with their corresponding length of stay in hours for diagnosis code 20 (cardiac patients) and Cluster Group 1 - see Figure 1. Cluster Group 1 was comprised of diagnosis codes 14, 24, 38, 35, 20, 25 and 26 (poisoning, central nervous system conditions - excluding strokes, diagnosis not classifiable, psychiatric conditions, cardiac conditions, respiratory conditions and gastrointestinal conditions respectively).

\begin{tabular}{lll}
\hline $\begin{array}{l}\text { Quantile } \\
(\%)\end{array}$ & $\begin{array}{l}\text { Diagnosis code 20 } \\
\text { Cardiac patients (hours) }\end{array}$ & $\begin{array}{l}\text { Cluster Group 1 } \\
\text { Diagnosis codes 14, 24, 38, 35, 20, } \\
25 \text { and 26 (hours) }\end{array}$ \\
\hline 0 & 0.02 & 0.01 \\
10 & 1.14 & 1.01 \\
20 & 1.56 & 1.41 \\
30 & 1.83 & 1.76 \\
40 & 2.23 & 2.10 \\
50 & 2.59 & 2.49 \\
60 & 3.09 & 2.91 \\
70 & 3.58 & 3.36 \\
80 & 3.91 & 3.83 \\
90 & 5.36 & 4.50 \\
100 & 14.76 & 16.06 \\
\hline
\end{tabular}

Table 2. 2004 ambulance attendance defined by their clustered group and the percentage of patients meeting the 4 hour target and the $98 \%$ target in hours. The 4 hour and $98 \%$ targets for the total A\&E (ambulance and walk-in arrivals) are shown for comparison.

\begin{tabular}{llll}
\hline 2004 Ambulance Arrivals (\%) & Cluster Group & $\%$ patients meeting 4 hour target & $98 \%$ target in hours \\
\hline $7,124(55.3 \%)$ & 1 & 89.13 & 8.16 \\
$535(4.2 \%)$ & 2 & 95.33 & 5.33 \\
$5,222(40.5 \%)$ & 3 & 93.28 & 5.94 \\
$12,881(100.0 \%)$ & 1,2 and 3 & 91.07 & 7.18 \\
\hline 47,018 (Total A\&E) & & 96.09 (Total A\&E) & 5.30 (Total A\&E) \\
\hline
\end{tabular}

Emergency Attendance Commissioning Data Sets (CDS Data) [9]. From the collected data, patients' arrival and discharge times (by day, hour and minute) and primary diagnosis codes were extracted. The length of stay (the difference between the arrival and discharge times) were calculated for each diagnosis code. Length of stay quantiles for each diagnosis code were extracted between $0 \%$ to $100 \%$ in steps of $10 \%$. Viewing the length of stay as quantiles showed the length of stay distribution of each diagnosis code. Hierarchical cluster analyses were performed on length of stay diagnosis quantiles to group together diagnoses with similar length of stay. The clustering method used was average linkage of euclidean distance [10], [11]. The clustering was limited to those diagnosis groups with 15 or more observations. Clustered diagnosis codes for the 2004 ambulance arrivals are shown in Figure 1. An example of the quantile data used to generate the cluster is shown in Table 1. In the CDS data, diagnosis code 20 referred to patients with cardiac conditions.

Observing Figure 1, at the height level of 4, an imaginary horizontal line intersects the vertical dendrogram lines identifying three clustered groups. The clustered groups were identified as follows: Cluster Group 1 (diagnosis codes 14, 24, 38, 35, 20, 25 and 26); Cluster Group 2 (diagnosis codes 16, 3, 17, 22, 10, 29, 31, 13, 12, 32 and 34); and Cluster Group 3 (diagnosis codes 39, 2, 6, 18, 30, 136, 5 and 27). Each of the three clustered groups had their diagnosis length of stay extracted to derive a length of stay profile for the whole clustered group.

With knowledge of the clusters and their diagnosis code associations, patient arrival data were extracted, matching patient arrival patterns to their clustered group. Therefore, for each clustered group, the length of stay distribution and patient arrival over a 24 hour, 7 day week period was determined. The model attached treatment places and nurse resources (if available) on patient arrival to A\&E. 


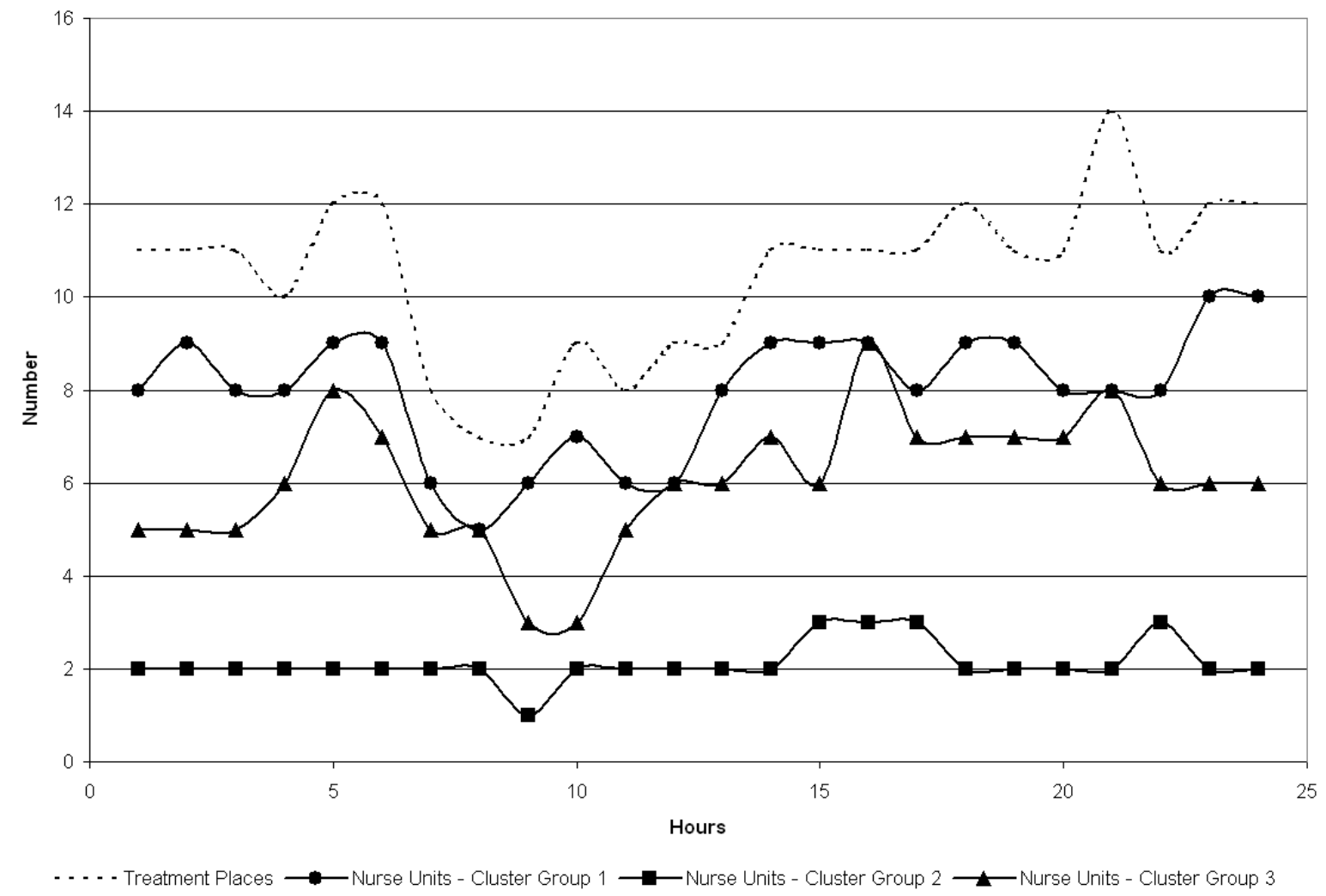

Figure 2. Shows the maximum number of patient treatment places resource and nurse unit resource required over a 52 week run of the model. Figure 2 shows the model run results with unconstrained resources to obtain the maximum number of resources required.

As nurses might be assigned to more than one patient, a fraction of a nurse (nurse units) was attached to a patient for the duration of their stay. The clustered length of stay distribution, their associated patient arrivals, treatment places and nurse resources acted as input parameters for the simulation model.

The allocation of nurses to patients was simply to assign nurses to patients' length of stay and made no attempt to qualify the nurse activity whilst allocated to a patient. For example, during a stay, a patient might require intensive nursing care or the patient might be waiting for other services requiring little actual nursing care. Similarly, no attempt was made to quantify similar clusters by treatment activity or intensity. Clustering was simply measured on the recorded length of stay. However, the clustering did appear to group together diagnosis codes that intuitively made sense. Within Cluster Group 1, diagnosis codes 14, 24, 38, 35, 20, 25 and 26 (poisoning, central nervous system conditions - excluding strokes, diagnosis not classifiable, psychiatric conditions, cardiac conditions, respiratory conditions and gastrointestinal con- ditions respectively) are very serious medical conditions often with extended periods of treatment and observations within A\&E. Cluster Group 3, captures diagnosis codes $39,2,6,18,30,1,36,5$ and 27 (nothing abnormal detected, contusions and abrasions, sprain and ligament injury, local infection, diabetes and other endocrinological conditions, lacerations, ophthalmological conditions, dislocations/fracture/joint injury/amputation and urological conditions respectively) containing many conditions that are treated without extended periods of observation. Cluster Group 2, capturing diagnosis codes 16, 3, 17, 22, 10 and 29 (visceral injury, soft tissue inflammation, infectious diseases, other vascular conditions, burns/scalds and gynaecological conditions respectively) also contains many conditions that are treated without extended periods of observation, but possibly nothing more serious than Cluster Group 3 . 


\section{Findings and Results}

Table 2 shows the ambulance attendance in 2004 defined by their clustered group. The percentage of patients meeting the 4 hour target and the $98 \%$ target in hours is also shown. Table 2 shows that Cluster Group 1 has the longest length of stay, followed by Cluster Group 3 and Cluster Group 2 respectively. Table 2 also shows that Cluster Group 1 and 3 had significantly more arrivals when compared to Cluster Group 2. None of the clustered groups met the $98 \% 4$ hour target in 2004. However, combining the ambulance arrivals with the walk-in arrivals, $96 \%$ of patients attending $\mathrm{A} \& \mathrm{E}$ met the 4 hour target.

Running the model with unconstrained resources over 52 weeks, Figure 2, showed the maximum number of patient treatment places resource and nurse units resource required. The results showed that the maximum number of ambulance arrival treatment places over the 52 week period was fourteen. The results also showed the mid morning period had the lowest levels of treatment places, suggesting a drop in ambulance arrival activity and an associated decrease in nurse units. The maximum number of the nurse units for Cluster Group 1, 2 and 3 was ten, three and nine respectively. For example, for Cluster Group 1 , ten nurse units, with a ratio of four patients to one nurse, would suggest two and a half nurses should be assigned for this group of patients. Though the results show resource use at hourly time intervals and could miss peaks or troughs occurring between hours, the information provided enables managers and clinicians to better understand and therefore better use health care resources. Data can also be extracted to determine statistical distribution of resources to improve understanding of regular activity.

\section{Summary}

In summary, this paper described a system for intelligent patient management in an A\&E setting using discrete-event simulation and clustering techniques. This paper shows how A\&E ambulance arrivals can be broken down into diagnosis sub-groups according to length of stay quantiles. The model also enabled the calculation of the maximum number of treatment places and nurse units required to service A\&E ambulance arrivals. Data input interfaces facilitate rapid data entry, as well as, input changes for scenario and sensitivity testing. Parameters to run the model can be extracted (with slight modification) from data currently collected from A\&E departments in England. The methodology described here can not only lead to significant improvement in patient management in A\&E departments, but could be generalised to other health care systems.

\section{References}

[1] Department of Health, "A\&E four hour total time target: Exceptions and performance ratings", 13 December 2003, http://www.dh.gov.uk/ assetRoot/04/07/00/47/04070047.PDF, Accessed 30 January 2006.

[2] S.C. Brailsford, V.A. Lattimer, P. Tarnaras and J.C. Turnbull, "Emergency and on-demand health care: modelling a large complex system", Journal of the Operational Research Society, 2004, 55, pp. 34-42.

[3] J.D. Griffiths, N. Price-Lloyd, M. Smithies and J.E. Williams, "Modelling the requirement for supplementary nurses in an intensive care unit", Journal of the Operational Research Society, 2005, 56, pp. 126-133.

[4] R. Ashton, L. Hague, M. Brandreth, D. Worthington and S. Cropper, "A simulation-based study of a NHS walk-in centre", Journal of the Operational Research Society, 2005, 56, pp. 153-161.

[5] A. Codrington-Virtue, P. Whittlestone, J. Kelly and T. Chaussalet "An interactive framework for developing simulation models of hospital accident and emergency services", Medical and Care Compunetics 2, IOS Press, Netherlands, 2005, 114, pp. 277-283.

[6] S. Walczak, W.E. Pofahl and R.J. Scorpio, "A decision support tool for allocating hospital bed resources and determining required acuity of care", Decision Support Systems, 2002, 34, pp. 445-456.

[7] M. W. Isken, and B. Rajagopalan, "Data mining to support simulation modeling of patient flow in hospitals", Journal of Medical Systems, 2002, 26, pp. 179-197.

[8] Simul8 Corporation, Simul8 Standard R11, http: // www. simul 8. com.

[9] NHS Data Model and Directory Service, "Commissioning Data Set (CDS) - Accident and Emergency Attendance CDS Type", http: //www.nhsia.nhs.uk/datastandards/ pages/ddm/data_dictionary/messages/ commissioning_data_set/accident_and_ emergency_attendance_cds_type_fr.asp, Accessed 30 January 2006.

[10] B.S. Everitt, and G. Dunn, "Applied multivariate data analysis - second edition", Arnold, London, 2001.

[11] W.N. Venables, and B.D. Ripley, "Modern applied statistics with S - fourth edition", Springer, New York, 2002. 